\section{Case Reports in Ophthalmology}

Case Rep Ophthalmol 2020;11:263-267

DOI: $10.1159 / 000508604$

Published online: July 3, 2020
(C) 2020 The Author(s)

Published by S. Karger AG, Basel www.karger.com/cop

This article is licensed under the Creative Commons Attribution-NonCommercial 4.0 International License (CC BY-NC) (http://www.karger.com/Services/OpenAccessLicense). Usage and distribution for commercial purposes requires written permission.

\title{
Corneal Opacification and Spontaneous Recovery following Injection of Healon5 into the Corneal Stroma during Intervention for Postoperative Hypotony
}

\author{
Michael Massengill ${ }^{a} \quad$ Charles Richard Blake ${ }^{b}$ \\ aUniversity of Florida College of Medicine, Gainesville, FL, USA; ${ }^{b}$ Department of \\ Ophthalmology, University of Florida, Gainesville, FL, USA
}

\section{Keywords}

Corneal opacification · Intrastromal injection · Healon $5 \cdot$ Sodium hyaluronate $\cdot$ Ocular hypotony

\begin{abstract}
We report the self-limited nature of corneal opacification after accidental injection of Healon5 into the corneal stroma. A 52-year-old male with a new diagnosis of severe stage, primary open-angle glaucoma underwent successful trabeculectomy OS, which was complicated by ocular hypotony and shallow anterior chamber (AC) on postoperative day 1 . Healon5, a hyaluronic acid-containing viscoelastic device, was accidentally introduced into the corneal stroma during attempted injection into the AC. The cornea hydrodissected and opacified, leading to precipitous loss of best-corrected visual acuity (BCVA). The corneal opacification spontaneously resolved over a 7-month period without specific intervention. During this period, the patient also underwent cataract extraction with posterior chamber intraocular lens placement and YAG capsulotomy, after which his BCVA returned to approximately baseline. Though intrastromal injection of sodium hyaluronate-containing material has been reported elsewhere, this complication with Healon5 use specifically has yet to be described in the literature and may occur in any procedure involving Healon5 in the AC. This case report is important, since
\end{abstract}




\section{Case Reports in Ophthalmology}

the precipitous loss of BCVA can be alarming to the ophthalmologist and the patient. The affected patient may be counseled that the opacification should improve with time.

(C) 2020 The Author(s)

Published by S. Karger AG, Basel

\section{Introduction}

Trabeculectomy and glaucoma drainage device implantation are performed to control intraocular pressure (IOP) in order to stymie glaucoma-disease progression. A complication that may occur after glaucoma surgeries is postoperative ocular hypotony that manifests as a shallow anterior chamber (AC) and, potentially, as vision loss via myopic and hyperopic shifts, hypotony maculopathy, and other pathophysiological mechanisms [1,2]. Thus, hypotonia may require intervention in the clinic. One method to temporarily treat ocular hypotony involves injection of an ophthalmic viscoelastic device (OVD) into the AC at the slit-lamp [3,4]. Healon $5{ }^{\mathrm{TM}}$, a viscoadaptive OVD that contains sodium hyaluronate at $2.3 \%$, can be employed to increase IOP and AC depth via intracameral injection. Healon 5 is commonly employed in other types of ophthalmic surgeries [5]. Here, we describe the self-limited natural history of accidental Healon5 injection into the corneal stroma during intracameral injection to treat postoperative hypotony after trabeculectomy.

\section{Case Report}

A 52-year-old male presented to the glaucoma service for evaluation of "blurry vision" OU and a "blind spot" OS. He was diagnosed with severe stage, primary open-angle glaucoma with cup-to-disc ratios of $1.0 \mathrm{OU}$, significant visual field loss OS greater than OD, IOPs of $14 \mathrm{~mm} \mathrm{Hg}$ (OS) and $13 \mathrm{~mm} \mathrm{Hg}$ (OD), and baseline best-corrected visual acuity (BCVA) of 20/40 OU. Due to his relatively young age and significant visual field loss OS, he underwent trabeculectomy OS with mitomycin C $300 \mu \mathrm{g} / \mathrm{mL}$ for 2 min without intraoperative complications.

He returned to the clinic on postoperative day 1 when his AC OS appeared extremely shallow with IOP measured at $3 \mathrm{~mm} \mathrm{Hg}$. To guard against hypotonic injury to the eye, Healon5, specifically selected in order to rapidly minimize the risk of choroidal hemorrhage and other complications, was to be injected into the $\mathrm{AC}$ at the slit-lamp rather than pursuing a conservative approach with atropine and steroids $[4,6]$. Since the eye of the patient was phakic and the $\mathrm{AC}$ was shallow, the plan included careful insertion of a 27 -gauge needle into the AC to a depth of the corneal thickness to avoid injury to the crystalline lens. However, the patient abruptly moved during the procedure as the Healon 5 was being ejected from the syringe, resulting in immediate hydrodissection and opacification of the cornea. It was then determined that the tip of the needle remained within the corneal stroma rather than the AC. The needle was subsequently re-inserted, this time with penetration into the $\mathrm{AC}$, and injection of Healon5 lead to improvement of IOP and AC depth.

As a result of Healon5-induced corneal opacification, the BCVA of the patient significantly decreased from baseline to light perception and hand motion immediately after the procedure, which persisted for the subsequent 1-month period (Fig. 1a). The cornea visibly cleared during months 2-7 after Healon5 injection, with concomitant improvement of the BCVA of the patient to between 20/100 and 20/400 (Fig. 1b, c). Given that he had developed a cortical and posterior subcapsular cataract OS, he then underwent cataract extraction with posterior chamber intraocular lens placement and subsequent YAG-capsulotomy for posterior capsular 


\section{Case Reports in Ophthalmology}

opacification that ultimately lead to a return to his baseline BCVA of 20/40 OS. His IOPs and cornea OS have remained stable and clear, respectively, through an 18-month period up to present following the injection of Healon5.

\section{Discussion}

Healon 5 is used in many ophthalmic procedures, including as a reagent to combat shallow $\mathrm{AC}$ and ocular hypotony after glaucoma surgery, as was employed for the patient described here [5]. Intracameral injection of Healon5 into the AC of a hypotonic eye aims to prevent complications that include choroidal detachment, hypotony maculopathy, and hyperopic or myopic shifts. Indeed, these complications were averted in our patient, including choroidal detachment, as the IOPs in our patient's eye stabilized with this intervention and time after trabeculectomy $[1,4,7]$. Nonetheless, we describe here a case where accidental injection of Healon5 into the corneal stroma during intracameral injection to mitigate ocular hypotony resulted in corneal hydrodissection and immediate opacification. The subsequent precipitous decline in BCVA may be alarming to both the ophthalmologist as well as the patient if the natural history of this accident is not known. Other documented complications [2] of Healon5 injection into the AC include spiking of IOP [8], endophthalmitis [9], and Descemet's membrane detachment [10].

To our knowledge, accidental injection of Healon5 into the corneal stroma has yet to be described in the literature. One case report described inadvertent intrastromal injection of Viscoat into the cornea of a pediatric patient during lensectomy for uveitic cataracts. In that case, injection occurred due to technical difficulties experienced during surgery when it became difficult to properly maneuver the tip of the OVD cannula into the AC. The injected Viscoat induced a relatively small area of opacification $(4 \mathrm{~mm}$ ) that cleared over a 3-month period with conservative management [11]. Furthermore, our literature search found that accidental intrastromal injection of OVD is more commonly associated with corneal opacification with concomitant detachment of Descemet's membrane, a pathology with an estimated recovery period of 6 weeks to 9 months with conservative management [12]. Of note, one such case report described corneal opacification after dispensing Healon GV into the corneal stroma when attempting to treat ocular hypotony after Ahmed valve implantation. Similar to our case, the patient moved suddenly during injection, causing needle misdirection and ectopic injection [12]. Taken together, to avoid accidental intrastromal injection of OVD, the ophthalmologist should vigilantly assess and correct for circumstances that may complicate complete insertion of the tip of the OVD cannula into the AC. To this end, providing optimal counseling prior to the procedure and analgesia during the procedure at the slit-lamp will help prevent patients from moving at the slit-lamp and avoid subsequent complications.

Intrastromal infiltration of OVD is a rare event and not easily studied in humans. Thus, specific interventions to enhance clearance of OVD from the cornea is lacking. Others have unsuccessfully attempted to hasten removal of OVD from the corneal stroma via stab incision and aspiration at the time of accidental injection or surgical irrigation and aspiration afterwards $[12,13]$. Thus, the prevailing treatment strategy, at present, is conservative management, as all reported cases of corneal opacification with or without Descemet's membrane detachment have resolved within 9 months [12].

The mechanisms by which sodium hyaluronate-containing OVD is removed endogenously by the cornea remains poorly understood. Others have suggested that defects in Descemet's membrane enable its efflux from the cornea into the AC [12]. In rabbits, infiltration 


\section{Case Reports in Ophthalmology}

Massengill and Blake: Spontaneous Recovery of Healon5-Induced Corneal Opacification

of the cornea by granulocytes and macrophages has also been posited to facilitate the removal of Healon from the cornea [13]. In general, reactive oxygen species (ROS) and hyaluronidases (HA) are known to play a role in the local degradation of hyaluronate in vivo and thus could facilitate degradation of OVD in the cornea [14]. From a clinical perspective, the rate of OVD removal may be dependent on the type of OVD employed (for example, Viscoat is of a smaller molecular weight and more dispersive compared to Healon5), the age of the patient and their metabolic rate, and the size of the OVD inoculation. The OVD degradation may also be dependent on the ocular inflammatory state, which could lead to local differences in $\mathrm{pH}, \mathrm{ROS}$, and HA expression [11, 12, 14]. Future studies in animal models could illuminate potential interventions to accelerate OVD metabolism in vivo. However, given the success of conservative management, such studies may not be prudent in terms of cost and potential side effects imposed by the intervention.

In conclusion, Healon5 is a commonly employed OVD in many ophthalmic procedures. Accidental injection of Healon5 into the corneal stroma results in self-limited corneal opacification that decreases BCVA in the short term. As in our case, improvement can be expected without significant long-term deleterious effects over the ensuing months.

\section{Acknowledgements}

The authors thank the staff of the University of Florida Ophthalmic Photography Facility for obtaining slit-lamp images of the presented patient.

\section{Statement of Ethics}

The authors obtained written informed consent from the patient to publish the medical history and slit-lamp photos in this case report.

\section{Disclosure Statement}

The authors report that they have no financial disclosures related to this publication.

\section{Funding Sources}

Supported by Training Grant F30EY027163 (awarded to M.M.).

\section{Author Contributions}

M.M. reviewed the medical charts, wrote the case report, and assembled the figure. C.R.B. treated the patient, reviewed the medical charts, stored and organized slit-lamp images, and edited the case report. The authors attest that they meet the requirements for authorship. 


\section{Case Reports in Ophthalmology}

\section{References}

1 Tunç Y, Tetikoglu M, Kara N, Sagdık HM, Özarpaci S, Elçioğlu MN. Management of hypotony and flat anterior chamber associated with glaucoma filtration surgery. Int J Ophthalmol. 2015 Oct;8(5):950-3.

2 Wang Q, Thau A, Levin AV, Lee D. Ocular hypotony: A comprehensive review. Surv Ophthalmol. 2019 Sep Oct;64(5):619-38.

3 Hoffman RS, Fine IH, Packer M. Stabilization of flat anterior chamber after trabeculectomy with Healon5. J Cataract Refract Surg. 2002 Apr;28(4):712-4.

4 Altangerel U, Rai S, Fontanarosa J, Moster MR. Intracameral 2.3\% sodium hyaluronate to treat postoperative hypotony in patients with glaucoma. Ophthalmic Surg Lasers Imaging. 2006 Mar-Apr;37(2):106-11.

5 Higashide T, Sugiyama K. Use of viscoelastic substance in ophthalmic surgery - focus on sodium hyaluronate. Clin Ophthalmol. 2008 Mar;2(1):21-30.

6 Tosi GM, Schiff W, Barile G, Yoshida N, Chang S. Management of severe hypotony with intravitreal injection of viscoelastic. Am J Ophthalmol. 2005 Nov;140(5):952-4.

7 Hosoda S, Yuki K, Ono T, Tsubota K. Ophthalmic viscoelastic device injection for the treatment of flat anterior chamber after trabeculectomy: a case series study. Clin Ophthalmol. 2013;7:1781-5.

8 Bruynseels A, Sii F, Masood I, Shah P. Severe Intraocular Pressure Elevation After Intracameral Healon 5 Viscoelastic Support for Postoperative Hypotony After XEN Gel Stent Insertion. J Glaucoma. 2018 Apr;27(4):e75-6.

9 Salvo EC Jr, Luntz MH, Medow NB. Use of viscoelastics post-trabeculectomy: a survey of members of the American Glaucoma Society. Ophthalmic Surg Lasers. 1999 Apr;30(4):271-5.

10 Rasouli M, Mather R, Tingey D. Descemet membrane detachment following viscoelastic injection for posttrabeculectomy hypotony. Can J Ophthalmol. 2008 Apr;43(2):254-5.

11 McConnell LK, Winter TW, Olson RJ, Longmuir SQ. Inadvertent intrastromal injection of ophthalmic viscoelastic. J AAPOS. 2013 Dec;17(6):639-41.

12 Chay E, Dastgir G, Scott W, Lazzaro D. Descemet's Membrane Dehiscence Resulting From Misdirected Viscoelastic During Anterior Chamber Reformation. Eye Contact Lens. 2018 Sep;44 Suppl 1:S355-7.

13 Hoover DL, Giangiacomo J, Benson RL. Descemet's membrane detachment by sodium hyaluronate. Arch Ophthalmol. 1985 Jun;103(6):805-8.

14 Fallacara A, Baldini E, Manfredini S, Vertuani S. Hyaluronic Acid in the Third Millennium. Polymers (Basel). 2018 Jun;10(7):E701.
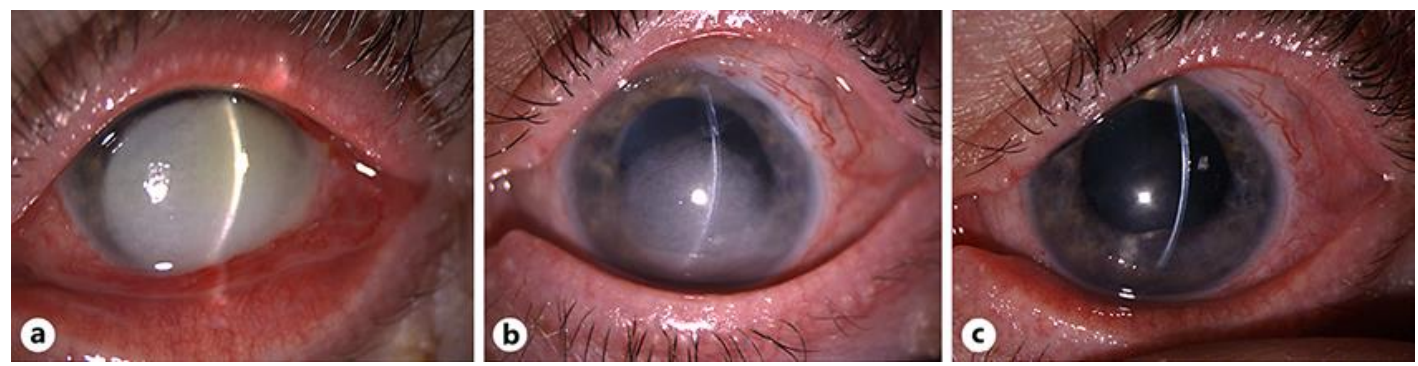

Fig. 1. Self-limited corneal opacification after accidental injection of Healon5 into the corneal stroma. a Near total corneal opacification shortly after injection. $\mathbf{b}$ The superior aspect of the cornea improved prior to near complete resolution over the ensuing months (c). The brightness of the images was modified to allow for comparison. 